\title{
Preoperative high-sensitivity C-reactive protein predicts depression in patients undergoing coronary artery bypass surgery: A single-center prospective observational study
}

\author{
Limeng Yang, MD, Jianyang Wang, MD, Lu Zhang, MD, Jianfeng Hou, MD, \\ Xin Yuan, MD, PhD, Shengshou $\mathrm{Hu}, \mathrm{MD}, \mathrm{PhD}$, and Zhe Zheng, MD, $\mathrm{PhD}$
}

\begin{abstract}
Objective: Our objective was to reveal the contribution of preoperative high-sensitivity C-reactive protein (hsCRP) levels to the risk of depression in patients undergoing coronary artery bypass grafting (CABG).
\end{abstract}

\begin{abstract}
Methods: In this study, 232 consecutive patients undergoing elective CABG were evaluated for depression utilizing the 9-item Patient Health Questionnaire scale at least 3 days before surgery and 6 months after CABG. In addition, peripheral blood samples were collected at baseline and the circulation levels of hsCRP were measured.
\end{abstract}

\begin{abstract}
Results: The preoperative and postoperative rate of depression was $18.1 \%$. Interestingly, preoperative depression was independently associated with women (odds ratio [OR], 2.49; 95\% confidence interval [CI], 1.13-5.50) and log-transformed hsCRP (OR, 1.16; 95\% CI, 1.06-1.27) after adjusting for various factors using logistic regression, including age, sex, and university-level education. Postoperative depression was only associated with $\log$-transformed hsCRP (OR, 1.15; 95\% CI, 1.05-1.25) in these patients. Log-transformed hsCRP remained associated with future depression after adjusting for postoperative medications or major adverse cardiovascular events during the follow-up period.
\end{abstract}

Conclusions: The present study is the first to report elevated serum hsCRP is an independent predictor for depression in CABG patients not only preoperatively but also up to 6 months after surgery. These clinical findings may reveal a potential target for improving prognosis in CABG patients with depression. (J Thorac Cardiovasc Surg 2012;144:500-5)

Depression is frequently observed in patients with coronary heart disease (CHD) ${ }^{1}$ and represents a significant risk factor for major cardiovascular events. ${ }^{2}$ Depression in patients with existing CHD confers a relative risk between 1.5 and 2.5 for cardiac morbidity and mortality. ${ }^{3}$ In previous cross-sectional and prospective studies, the prevalence of major depression in patients with $\mathrm{CHD}$ ranged from $10 \%$ to $47 \%,{ }^{4}$ and slightly higher rates were reported $(28 \%)$ in patients requiring coronary artery bypass grafting (CABG). ${ }^{5}$ In CABG patients, symptoms of depression may

From the Department of Cardiovascular Surgery, State Key Laboratory of Translational Cardiovascular Medicine, Fuwai Hospital \& Cardiovascular Institute, Chinese Academy of Medical Sciences, Peking Union Medical College, Beijing, China.

Funding: The study was supported by Basic Scientific Research Fund of National Public Scientific Institute, Program for Changjiang Scholars and Innovative Research Team in University, Ministry of Health Industry Special Foundation, and Ministry of Education New Century Foundation for Outstanding Scholarship.

Disclosures: Authors have nothing to disclose with regard to commercial support.

Ethics approval: The study was approved by the ethics committee of Fuwai Cardiovascular Hospital and all patients gave the written informed consent.

L.Y., J.W., and L.Z. contributed equally to this work.

Received for publication July 28, 2011; revisions received Nov 18, 2011; accepted for publication Jan 6, 2012; available ahead of print Feb 6, 2012.

Address for reprints: Zhe Zheng, MD, PhD, 167 Beilishi Rd, Xi Chen District, Beijing, China, 100037 (E-mail: zhengzhefuwai@tom.com).

$0022-5223 / \$ 36.00$

Copyright (c) 2012 by The American Association for Thoracic Surgery doi:10.1016/j.jtcvs.2012.01.034 increase mortality after bypass surgery ${ }^{5}$ and were associated with atherosclerotic progression. ${ }^{6}$ In 2008, the American Heart Association recommended routine screening for depression in patients with CHD in various settings, including the hospital, physician's office, clinic, and cardiac rehabilitation center. ${ }^{7}$

In recent years, growing evidence also suggests the existence of a bidirectional relationship between depressed mood and inflammation. ${ }^{8,9}$ Dantzer and associates ${ }^{10}(2008)$ reported that inflammation can lead to the development of symptoms of depression in vulnerable individuals. Consistent with these findings, high-sensitivity C-reactive protein (hsCRP), currently as one available clinical biomarker of inflammation, was reported associated with increased long-term mortality and extended hospital length of stay in patients undergoing nonemergency CABG-only surgery. ${ }^{11}$ The latest research has proved the association between depression and C-reactive protein in healthy adults. ${ }^{12}$ However, the contribution of hsCRP to depression remains poorly understood in patients requiring CABG. To date, previous studies reported several risk factors for depression in CABG patients, including age, ${ }^{13}$ female gender, ${ }^{14}$ and education. ${ }^{15}$ Therefore, the primary purpose of our study was to investigate the predictive value of preoperative hsCRP as a risk marker for major depression in $\mathrm{CABG}$ patients before surgery and 6 months after CABG. 


\section{Abbreviations and Acronyms \\ $\mathrm{CABG}=$ coronary artery bypass grafting \\ $\mathrm{CHD}=$ coronary heart disease \\ CI $=$ confidence interval \\ hsCRP = high-sensitivity C-reactive protein \\ In-hsCRP $=$ log-transformed high-sensitivity \\ C-reactive protein \\ MACE = major adverse cardiac event \\ $\mathrm{OR}=$ odds ratio \\ PHQ = Patient Health Questionnaire scale}

\section{METHODS}

\section{Study Population}

This study was designed as a prospective observational study to identify the contribution of blood hsCRP to depressive symptoms among patients undergoing $\mathrm{CABG}$ surgery. Therefore, the data were prospectively collected from a consecutive series of patients undergoing their first bypass operation at Fuwai Hospital in Beijing, China. In brief, 258 consecutive patients who underwent first-time elective CABG from March 2010 to September 2010 at Fuwai Hospital were approached to participate in this study. The exclusion criteria included the following subjects: (1) less than 18 years of age, (2) subjects with previous CABG, and (3) a language barrier preventing questionnaire completion. The remaining cohort of 234 patients who underwent first-time elective CABG was eligible for the study. Of them, 2 patients died during hospitalization. This study was approved by the local ethics committee, and all participants of the study were voluntary and provided written informed consent.

Each participant completed the baseline evaluation at least 3 days before CABG, including depressive symptoms, medical history, physical examination, use of medications, and collection of fasting blood samples. Additional information was collected by telephone 6 months later, including postoperative medications (aspirin, $\beta$-blockers, or statin use) and major adverse cardiac events (MACEs: all-cause death, nonfatal myocardial infarction, and any target-vessel revascularization). In addition, depressive symptoms were assessed by the 9-item Patient Health Questionnaire scale (PHQ) at baseline and 6 months after surgery. At last, 232 participants completed the whole study with no patients lost during the follow-up period.

\section{Assessment of Depressive Symptoms}

Depressive symptoms were assessed with the 9-item $\mathrm{PHQ}^{16}$ during hospitalization at least 3 days before $\mathrm{CABG}$, as well as 6 months after surgery via telephone contact. PHQ-9 is a 9-item self-administered instrument to assess depressive symptoms over the past 2 weeks that maps 9 symptoms from the Diagnostic and Statistical Manual of Mental Disorders, edition 4 , classification for major depressive disorder. ${ }^{16}$ Previous studies have established PHQ-9 as a valid tool for depression screening ${ }^{17}$ and it seems reliable and valid for detecting major depressive disorder in Chinese patients. ${ }^{18}$ Telephone administration of the PHQ-9 appeared to generate a reliable procedure for assessing depression. ${ }^{19}$ PHQ-9 score provides a continuous score of depressive symptom severity range from 0 to 27 . Patients had to score 10 or more, indicating a moderate to severe level of depressive symptoms. For this study, we chose PHQ-9 of 10 or more as the screening cutoff point for the diagnosis of major depression, inasmuch as a PHQ-9 score of at least 10 had a sensitivity and specificity of $88 \%$ for a clinical diagnosis of major depression. ${ }^{17}$

\section{Measurement of hsCRP}

Blood samples collected at baseline were stored at $-80^{\circ} \mathrm{C}$ until protein analyses were performed. The circulation levels of hsCRP were assessed by the high-sensitivity nephelometric method (Dade Behring Marburg $\mathrm{GmbH}$, Marburg, Germany) that displays a detection limit of $0.1 \mathrm{mg} / \mathrm{L}$. The intra-assay precision (coefficient of variation) was less than $5.5 \%$ and the interassay coefficient of variation was less than $8.2 \%$ in our laboratory.

\section{Statistical Analysis}

For descriptive purpose, categorical data were expressed as frequencies and continuous data are reported as mean and standard deviation (mean \pm standard deviation). The continuous variables and frequencies between groups were compared by the Student $t$ test and $\chi^{2}$ or Fisher exact test. Owing to the skewed distribution of hsCRP, it was log transformed for analyses. Binary logistic regression was used to estimate the odds ratio (OR) of log-transformed hsCRP (In-hsCRP) to the major depression in patients undergoing CABG. ${ }^{17}$ We successfully adjusted demographic data (age, gender) and the possible confounding effects of some social characteristics (university-level education), obesity, clinical characteristics (diabetes, hypertension, chronic pulmonary disease, valve lesion, cerebrovascular accident, and recent myocardial infarction), cardiac functional status (New York heart Association classes, left ventricular ejection fraction), and statin use. The aforementioned 13 adjustment factors include the following: the factors that had been revealed in previous studies-age, ${ }^{13}$ gender, ${ }^{14}$ university-level education, ${ }^{15}$ obesity ${ }^{20}$ - and other factors that indicated the disease severity of patients-clinical characteristics and aforementioned cardiac functional status, that is, preoperative statin use considering the effect of statins on hsCRP levels. ${ }^{21}$ For the follow-up results, MACE as a potential risk factor of major depression was added into the multivariate analysis model. The optimal cutoff value of hsCRP for major depression with the combination of the highest sensitivity and specificity was obtained by receiver operating characteristic curve. All tests for statistical significance were 2-tailed with $\alpha=.05$. All analyses were performed with SPSS Package for Windows version 13.0 (SPSS, Inc, Chicago, Ill).

\section{RESULTS}

\section{Baseline Characteristics}

In this study, 232 patients completed all questionnaires at baseline and up to 6 months after CABG surgery (Figure 1). No patients died or were lost during the 6-month follow-up questionnaire. The mean age of these patients was $60.8 \pm 8.6$ years, and the majority of the patients were male $(81.5 \%)$. Among the patients, 155 $(66.8 \%)$ had a history of hypertension, and $67(28.9 \%)$ had diabetes mellitus. Additionally, 104 (44.8\%) patients

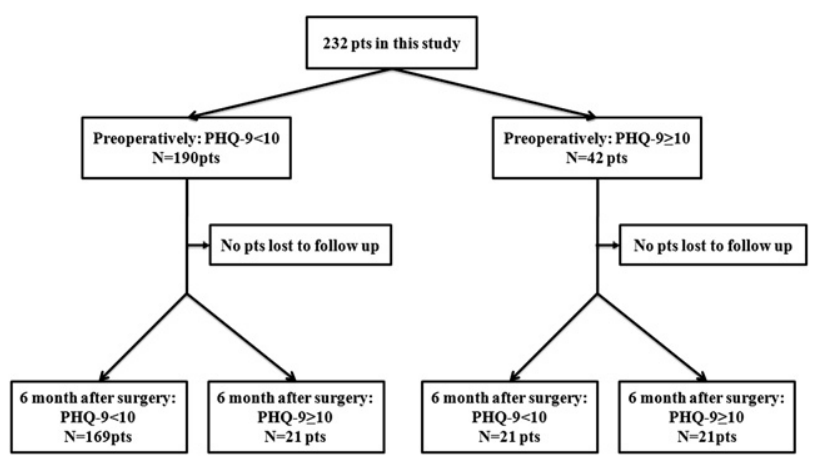

FIGURE 1. Participants available for analysis from the study. $P H Q, \mathrm{~Pa}-$ tient Health Questionnaire scale. 
exhibited recent myocardial infarction during the 3-week period before the operation, and $28(12.1 \%)$ patients also had valve disease. Of the participants, 38.8\% (90/232) were treated with statin medication more than 1 month before surgery, a rate that was higher than previously reported. ${ }^{8}$ Compared with nondepressed patients, depressed patients were more often female, had a low education level, and had a cerebrovascular accident or valve lesion (Table 1).

\section{Depression Evaluation Outcomes}

PHQ-9 scores ranged from 0 to 21 preoperatively and from 0 to 23 at follow-up assessment. The mean value of PHQ-9 scores was $4.8 \pm 5.0$ at baseline and $4.2 \pm 5.0$ at 6 months after surgery. A total of $42(18.1 \%)$ had major depression (PHQ-9 $\geq 10$ ) preoperatively. During the 6-month follow-up questionnaire, $42(18.1 \%)$ fulfilled the diagnosis of major depressive disorder. Likewise, we found a higher frequency of depressive symptoms in women than men both preoperatively $(30.2 \%$ vs $15.3 \% ; P=.022)$ and postoperatively $(20.9 \%$ vs $17.5 \% ; P=.594)$. Although the occurrence of depressive symptoms was similar preoperatively and postoperatively, we observed obvious changes in individuals (Figure 2). No significant depressive symptoms (PHQ-9 < 10) before or after surgery were found in $72.7 \%$ (169/232). New-onset depression occurred in $9.1 \%(21 / 232)$, transient depression in $9.1 \%(21 / 232)$, and persistent depression in $9.1 \%$ (21/232). The levels of hsCRP are shown in Figure 3. Results indicated that significant difference in hsCRP levels were observed in patients with the ollowing 4 depression status: never depression, new-onset depression, transient depression, and persistent depression.

\section{Postoperative Medications and MACE in CABG Patients With and Without Depression at 6 Months' Follow-up}

Table 2 presents the postoperative medications and the MACE rate in patients with and without depression during the 6-month follow-up. None of the patients used selective serotonin reuptake inhibitors at all during the study. No significant difference was detected in postoperative medications between depressed patients and nondepressed patients including aspirin, $\beta$-blockers, and statins. The incidence of MACE was $4.3 \%$. Compared with nondepressed patients, depressed patients displayed higher rates of nonfatal myocardial infarction $(0.0 \%$ vs $7.1 \% ; P=.006)$, any target-vessel revascularization $(0.0 \%$ vs $4.8 \% ; P=.032)$, and MACE $(2.6 \%$ vs $11.9 \% ; P=.024)$.

In all participants, univariate analysis demonstrated an association between depression symptoms and an increased incidence of MACE (Table 2). However, addition of MACE in model 2 (Table 3) results indicated that the contribution of MACE to postoperative depression was not observed in CABG patients (OR, 4.46; 95\% confidence interval [CI], 0.89-22.37).

\section{Contribution of hsCRP to Depression}

We investigated the contribution of hsCRP to the risk of depressive symptoms, and the results of the regression analysis are presented in Table 3. Multivariate analysis revealed that each standard deviation increase in In-hsCRP was associated with an increased OR for depression (preoperatively: OR, $1.16 ; P=.001 ; 6$ months' follow-up: OR, 1.15 ; $P=.002$ ), and hsCRP was correlated with depression both preoperatively and 6 months after surgery. Considering the possible influence of adverse cardiac events and

TABLE 1. Characteristics of CABG patients with and without major depression

\begin{tabular}{|c|c|c|c|c|c|c|}
\hline \multirow[b]{2}{*}{ Variables } & \multicolumn{3}{|c|}{ Preoperative depression } & \multicolumn{3}{|c|}{ Postoperative depression } \\
\hline & No $(n=190)$ & Yes $(n=42)$ & $P$ value & No $(n=190)$ & Yes $(n=42)$ & $P$ value \\
\hline Age, y & $60.4 \pm 8.4$ & $62.5 \pm 9.1$ & .166 & $60.9 \pm 8.4$ & $60.2 \pm 9.5$ & .643 \\
\hline Female, $\%$ & 15.8 & 31.0 & $.022 \ddagger$ & 17.9 & 21.4 & .594 \\
\hline University-level education, $\%$ & 18.9 & 17.1 & .780 & 20.0 & 11.9 & .222 \\
\hline BMI, $\mathrm{kg} / \mathrm{m}^{2}$ & $25.5 \pm 2.8$ & $25.7 \pm 3.0$ & .580 & $25.5 \pm 2.8$ & $25.7 \pm 3.0$ & .710 \\
\hline Diabetes mellitus, \% & 29.5 & 26.2 & .671 & 29.5 & 26.2 & .671 \\
\hline Hypertension, \% & 65.3 & 73.8 & .287 & 67.9 & 61.9 & .456 \\
\hline Chronic pulmonary disease, $\%$ & 2.1 & 0.0 & 1.000 & 1.6 & 2.4 & .553 \\
\hline Valve lesion, $\%$ & 10.5 & 19.0 & .125 & 11.6 & 14.3 & .626 \\
\hline $\mathrm{CVA}, \%$ & 11.1 & 11.9 & 1.000 & 14.3 & 20.6 & .485 \\
\hline Recent MI* & 44.7 & 45.2 & .953 & 43.7 & 50.0 & .456 \\
\hline NYHA classes I/II/III, \% & $9.5 / 81.6 / 8.9$ & $4.8 / 76.2 / 19.0$ & .114 & $7.4 / 81.6 / 11.0$ & $14.3 / 76.2 / 9.5$ & .349 \\
\hline LVEF, \% & $59.4 \pm 9.4$ & $57.8 \pm 9.4$ & .310 & $59.3 \pm 9.1$ & $58.2 \pm 10.7$ & .467 \\
\hline Statin use, $\% \dagger$ & 36.3 & 50.0 & .100 & 37.9 & 42.9 & .550 \\
\hline hsCRP, mg/L & $3.0 \pm 2.8$ & $5.0 \pm 5.2$ & $.021 \ddagger$ & $3.0 \pm 3.0$ & $4.9 \pm 4.7$ & $.015 \ddagger$ \\
\hline
\end{tabular}

Data are expressed as means \pm standard deviation. $C A B G$, Coronary artery bypass grafting; $B M I$, body mass index; $C V A$, cerebrovascular accident; $M I$, myocardial infarction; $N Y H A$, New York Heart Association; $L V E F$, left ventricular ejection fraction; $h s C R P$, high-sensitivity C-reactive protein. *Three weeks before surgery. $\dagger$ Regular application of statins more than 1 month before surgery. $\ddagger P<.05$. 


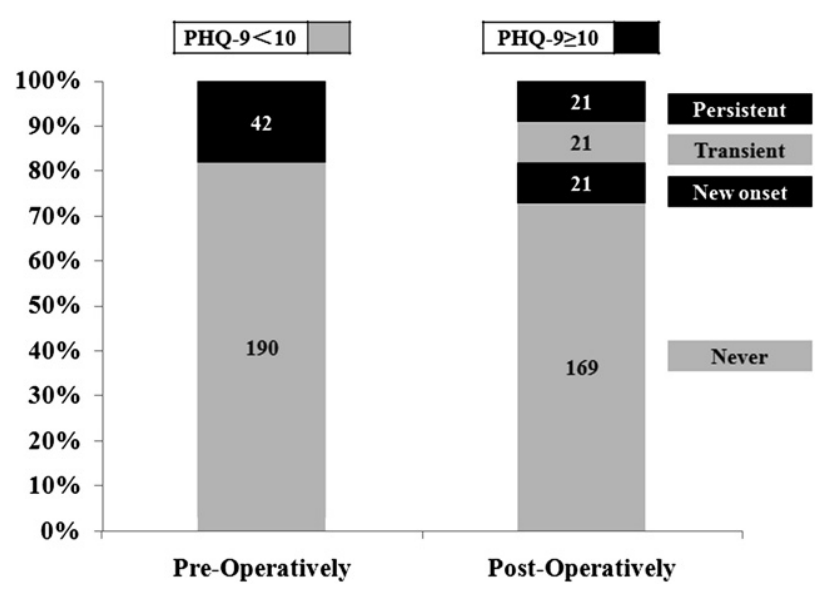

FIGURE 2. Numbers of patients with depression at baseline and 6 months after bypass surgery. The gray bars indicate the numbers of patients with a PHQ-9 score lessthan 10; the black bars indicate the number of patients with depression defined by a PHQ- 9 score of 10 or more. Labels on the right show the different classifications based on depressive symptoms preoperatively and postoperatively-never, new onset, transient, and persistent. $P H Q$, Patient Health Questionnaire scale.

postoperative medications on postoperative depression, we also additionally adjusted for postoperative medications (aspirin, $\beta$-blockers, and statin use) and MACE in model 2 . We obtained similar results, concluding that the InhsCRP (OR. 1.14; 95\% CI, 1.04-1.25) remained an independent predictor for postoperative major depression.

Further analyses were done, and the baseline depressive symptoms (PHQ-9 scores) were added into model 2 (Table 3). Results showed that baseline PHQ-9 scores were significantly associated with the postoperative depression (OR, 1.09; 95\% CI, 1.06-1.35). When the 2 risk factors baseline PHQ-9 and hsCRP were put into the same model (model 2), both PHQ-9 scores (OR, 1.04; 95\% CI, 1.01-1.17) and hsCRP (OR, 1.13; 95\% CI, 1.05-1.25)

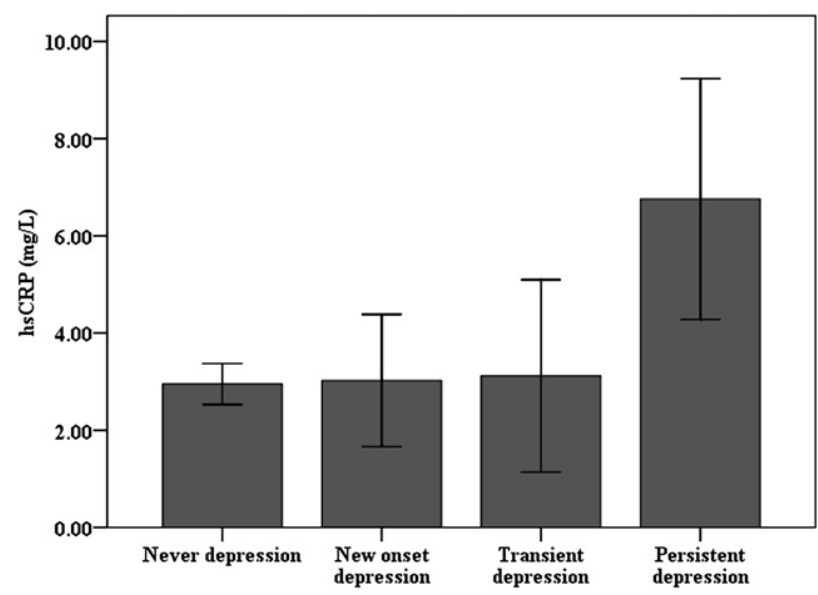

FIGURE 3. High-sensitivity C-reactive (hSCRP) protein levels in patients with 1 of the 4 stages of depression.
TABLE 2. Postoperative medications and MACE in patients with and without depression at 6 months after CABG

\begin{tabular}{lccc}
\hline & \multicolumn{2}{c}{ Postoperative depression } & \\
\cline { 2 - 3 } & $\begin{array}{c}\text { No }(\%) \\
(\mathbf{n}=\mathbf{1 9 0})\end{array}$ & $\begin{array}{c}\text { Yes (\%) } \\
(\mathbf{n}=\mathbf{4 2})\end{array}$ & $\boldsymbol{P}$ value \\
\hline Postoperative medications & & & \\
$\quad$ Aspirin & 76.8 & 76.2 & .928 \\
$\quad \beta$-blockers & 87.9 & 88.1 & .971 \\
$\quad$ Statins & 16.3 & 19.5 & .668 \\
Adverse cardiac events & & & \\
$\quad$ All cause death & 0.0 & 0.0 & - \\
$\quad$ Nonfatal MI & 0.0 & 7.1 & $.006^{*}$ \\
$\quad$ Target-vessel & 0.0 & 4.8 & $.032^{*}$ \\
$\quad$ revascularization & & & \\
$\quad$ MACE & 2.6 & 11.9 & $.024^{*}$ \\
\hline
\end{tabular}

MACE (major adverse cardiac event) is defined as all-cause death, nonfatal myocardial infarction $(M I)$, or any target-vessel revascularization (percutaneous coronary intervention and/or coronary artery bypass grafting $[C A B G]) .{ }^{*} P<.05$.

were independently associated with the postoperative depression.

Preoperative elevations in hsCRP levels were analyzed as a continuous variable as a risk predictor for depression in this study. In our study, the best cutoff point was not obtained through the receiver operating characteristic curve, and we observed that participants with higher levels of hsCRP had more serious depressive symptoms. The area under the receiver operating characteristic curve for hsCRP in the prediction of depression preoperatively and postoperatively is presented in Table 4. The analysis indicated that the predictive function of hsCRP for preoperative depression is best in patients with an hsCRP level more than $2.5 \mathrm{mg} / \mathrm{L}$ (area under the curve $=0.795$ ); thereby, this analysis is excellent in patients with an hsCRP level of $3.0 \mathrm{mg} / \mathrm{L}$ or more (area under the curve $=0.704$ ) for postoperative depressive symptoms.

Considering the anti-inflammatory effects of drugs, statins were treated as a potential confounder in this study. ${ }^{21}$ Consistent with the pharmacokinetic principle, preoperative statin use was defined as preoperative regular application of statins for more than 1 month. A high rate

TABLE 3. Multivariable-adjusted OR of depression associated with hsCRP

\begin{tabular}{llcc}
\hline & \multicolumn{3}{c}{ Regression model, OR $(\mathbf{9 5} \%$ CI) } \\
\cline { 2 - 4 } & \multicolumn{1}{c}{ Base model } & Model 1 & Model 2 \\
\hline Preop & $1.15(1.06-1.26)$ & $1.15(1.06-1.26)$ & $1.16(1.06-1.27)$ \\
Postop & $1.26(1.08-1.47)$ & $1.15(1.05-1.25)$ & $1.15(1.05-1.25)$ \\
\hline Base model was adjusted for age and gender. Model 1 was adjusted for the variables in \\
base model plus social risk factors including university-level education. Model 2 was \\
adjusted for all variables in model 1 plus obesity (body mass index > 30.0), diabetes, \\
hypertension, chronic pulmonary disease, valve lesion, cerebrovascular accident, \\
New York Heart Association functional classification, left ventricular ejection \\
fraction, and statin use. High-sensitivity C-reactive protein was log transformed for \\
analysis. OR, Odds ratio; hsCRP, high-sensitivity C-reactive protein; $C I$, confidence \\
interval.
\end{tabular}


TABLE 4. hsCRP predict depression for CABG patients with different hsCRP levels

\begin{tabular}{|c|c|c|c|c|c|c|c|c|c|c|c|}
\hline & & \multicolumn{10}{|c|}{ hsCRP levels (mg/L) } \\
\hline & & $<\mathbf{1 . 0}$ & $\geq \mathbf{1 . 0}$ & $<1.5$ & $\geq 1.5$ & $<\mathbf{2 . 0}$ & $\geq \mathbf{2 . 0}$ & $<2.5$ & $\geq 2.5$ & $<\mathbf{3 . 0}$ & $\geq \mathbf{3 . 0}$ \\
\hline \multirow{3}{*}{ Area under ROC } & $\mathbf{n}$ & 43 & 189 & 90 & 142 & 112 & 120 & 137 & 95 & 153 & 79 \\
\hline & Preop & 0.482 & 0.582 & 0.480 & 0.630 & 0.539 & 0.763 & 0.463 & 0.795 & 0.406 & 0.742 \\
\hline & Postop & 0.425 & 0.621 & 0.531 & 0.673 & 0.482 & 0.656 & 0.489 & 0.698 & 0.481 & 0.704 \\
\hline
\end{tabular}

hsCRP, High-sensitivity C-reactive protein; $C A B G$, coronary artery bypass grafting; $R O C$, receiver operating characteristic

of preoperative statin use was observed in our study population (38.8\% at baseline). Univariate analysis indicated no significant difference in statin use among the depressed patients and those without depression preoperatively $(50.0 \%$ vs $36.3 \% ; P=.100)$ and postoperatively $(19.5 \%$ vs $16.3 \% ; P=.668)$. Furthermore, multivariate analysis showed that preoperative and postoperative statin medication use was not associated with depressive mood in CABG patients (model 2, preoperatively: OR, 1.82; 95\% CI, 0.88-3.78; $P=.106$; postoperatively: OR, $1.34 ; 95 \% \mathrm{CI}, 0.65-2.76 ; P=.433$ ). Inasmuch as none of our study participants with depressive symptoms used selective serotonin reuptake inhibitors during this whole study, we did not adjust for selective serotonin reuptake inhibitor antidepressants as a confounding factor in the study.

\section{DISCUSSION}

The present prospective study evaluated the predictive value of preoperative circulation hsCRP for major depression in patients undergoing CABG. A direct relationship was observed in that the higher hsCRP levels were associated with a higher risk of having major depression for CABG patients. Data from our study demonstrated that preoperative depression was associated with an increased risk ranging from $15 \%$ to $16 \%$ for each standard deviation increase in In-hsCRP. For postoperative depression the increased risk ranged from $15 \%$ to $26 \%$ in this group as described in Table 3.

These findings also suggest that inflammation may play an important role in the pathophysiology of depression in CABG patients. There were some other psychophysiologic factors that had been revealed in previous studies such as autonomic nervous system ${ }^{22}$ and serotonin metabolism. ${ }^{23}$ Although the bidirectional association between depression and cardiovascular disease is widely documented, as both depression and CHD are heterogeneous diseases, it is unlikely that a single psychophysiologic factor will explain a substantial proportion of the prospective association between depression and CHD owing to the complexity of the network of systems involved. ${ }^{24}$ Further study will be needed to explore the role of hsCRP in the pathophysiologic mechanisms of major depression and confirm whether hsCRP can serve as a therapeutic target for depression in CABG patients.
To date, depression as a major risk factor for patients after CABG surgery had been consistent in the literature. ${ }^{6}$ Likewise, risk factors for depression such as age, ${ }^{13}$ gender, ${ }^{14}$ university-level education, ${ }^{15}$ and obesity ${ }^{20}$ were confirmed in this previous research. Although some studies had explored the relationship between hsCRP and major depression in patients with CHD, these studies did not adjust for the recognized risk factors mentioned herein. Therefore, our study clearly assessed the prediction function of hsCRP for depression among CABG patients using logistic regression models.

In previous studies, ${ }^{8}$ the utilization rate of statins was lower in the study participants than in our patients. We believe the possible reason may be the inherent differences between the CHD patients and CABG patients, who usually had more serious lesions in the coronary arteries than is common for patients with CHD. However, we get similar results that the proportion of patients treated with statins tends to be higher in patients with depressive symptoms (Tables 1 and 2).

In this study, as the primary study objective of our study, we did not further explore the contribution of depression to MACE in CABG patients. However, considering the potential effect of MACE on postoperative depression, we also added MACE as a confounding factor in model 2, and analysis showed that it did not change the result. Furthermore, our finds indicated that the risk of MACE in CABG patients with preoperative major depression should be discussed when obtaining the patient's informed consent by cardiac surgeons and postoperative follow-up was needed. Depression recognition and management may improve outcomes in these patients.

As mentioned earlier, both hsCRP and depression are significant risk factors of poor outcomes for patients undergoing $\mathrm{CABG}{ }^{5,11}$ Our findings have proved the independent association between the 2 factors. Further study will be needed to examine whether hsCRP can serve as a therapeutic target for depression in CABG patients. If the results are positive, it would be of great clinical significance for the improvement of outcomes in depressed patients undergoing bypass surgery.

\section{Limitations}

There are potential limitations to our study. First, we could not obtain biochemical results from these participants 
inasmuch as the follow-up was completed via telephone contact. Therefore, future studies may be required to explore the association between the continued changes of inflammatory proteins and the severity of depressive symptoms in CABG patients. Second, we investigated only a single measure of systemic inflammation, and some other possible associated inflammatory markers such as interleukin-6, tumor necrosis factor- $\alpha$, and interferon- $\gamma^{25}$ were not included in this study. Another limitation of our study is that it is a single-center study. Although this study was conducted at a single center, Fuwai Hospital, the participants of this study were from several different areas of China, so no regional selection bias existed in this research. Nonetheless, the present study designed with a prospective observation method could exclude the effect of possible selection biases.

\section{CONCLUSIONS}

Our study suggests that hsCRP displays a consistent and significant prognostic value for major depression in CABG patients both preoperatively and 6 months after surgery. These findings could ultimately be used to improve the prognosis of depressed patients after CABG.

We acknowledge the participants in this study as well as the physicians, nurses, and administrative staff at Fuwai Hospital who assisted with various aspects of this study. We also thank $\mathrm{Li}$ He and Huan Ren for their tireless efforts in blood sample collection.

\section{References}

1. Blumenthal JA. Depression and coronary heart disease: association and implications for treatment. Cleve Clin J Med. 2008;75(Suppl 2):S48-53.

2. Whooley MA, de Jonge P, Vittinghoff E, Otte C, Moos R, Carney RM, et al. Depressive symptoms, health behaviors, and risk of cardiovascular events in patients with coronary heart disease. JAMA. 2008;300:2379-88.

3. Lett HS, Blumenthal JA, Babyak MA, Sherwood A, Strauman T, Robins C, et al. Depression as a risk factor for coronary artery disease: evidence, mechanisms, and treatment. Psychosom Med. 2004;66:305-15.

4. Thombs BD, Bass EB, Ford DE, Stewart KJ, Tsilidis KK, Patel U, et al. Prevalence of depression in survivors of acute myocardial infarction. $J$ Gen Intern Med. 2006;21:30-8

5. Connerney I, Sloan RP, Shapiro PA, Bagiella E, Seckman C. Depression is associated with increased mortality 10 years after coronary artery bypass surgery. Psychosom Med. 2010;72:874-81.

6. Wellenius GA, Mukamal KJ, Kulshreshtha A, Asonganyi S, Mittleman MA. Depressive symptoms and the risk of atherosclerotic progression among patients with coronary artery bypass grafts. Circulation. 2008;117:2313-9.

7. Lichtman JH, Bigger JT Jr, Blumenthal JA, Frasure-Smith N, Kaufmann PG, Lespérance F, et al, American Heart Association Prevention Committee of the Council on Cardiovascular Nursing; American Heart Association Council on Clinical Cardiology; American Heart Association Council on Epidemiology and Prevention; American Heart Association Interdisciplinary Council on Quality of Care and Outcomes Research; American Psychiatric Association.
Depression and coronary heart disease: recommendations for screening, referral, and treatment: a science advisory from the American Heart Association Prevention Committee of the Council on Cardiovascular Nursing, Council on Clinical Cardiology, Council on Epidemiology and Prevention, and Interdisciplinary Council on Quality of Care and Outcomes Research: endorsed by the American Psychiatric Association. Circulation. 2008;118:1768-75.

8. Empana JP, Sykes DH, Luc G, Juhan-Vague I, Arveiler D, Ferrieres J, et al. Contributions of depressive mood and circulating inflammatory markers to coronary heart disease in healthy European men: the Prospective Epidemiological Study of Myocardial Infarction (PRIME). Circulation. 2005;111:2299-305.

9. Stewart JC, Rand KL, Muldoon MF, Kamarck TW. A prospective evaluation of the directionality of the depression-inflammation relationship. Brain Behav Immun. 2009;23:936-44.

10. Dantzer R, O'Connor JC, Freund GG, Johnson RW, Kelley KW. From inflammation to sickness and depression: when the immune system subjugates the brain Nat Rev Neurosci. 2008;9:46-56.

11. Perry TE, Muehlschlegel JD, Liu KY, Fox AA, Collard CD, Body SC, et al., CABG Genomics Investigators. Preoperative $\mathrm{C}$-reactive protein predicts longterm mortality and hospital length of stay after primary, nonemergent coronary artery bypass grafting. Anesthesiology. 2010;112:607-13.

12. Ma Y, Chiriboga DE, Pagoto SL, Rosal MC, Li W, Merriam PA, et al. Association between depression and C-reactive protein. Cardiol Res Pract. 2010;2011: 286509.

13. Krannich JH, Weyers P, Lueger S, Herzog M, Bohrer T, Elert O. Presence of depression and anxiety before and after coronary artery bypass graft surgery and their relationship to age. BMC Psychiatry. 2007;7:47.

14. Naqvi TZ, Rafique AM, Andreas V, Rahban M, Mirocha J, Naqvi SS. Predictors of depressive symptoms post-acute coronary syndrome. Gend Med. 2007;4: 339-51.

15. Dunkel A, Kendel F, Lehmkuhl E, Babitsch B, Oertelt-Prigione S, Hetzer R, et al Predictors of preoperative depressive risk in patients undergoing coronary artery bypass graft surgery. Clin Res Cardiol. 2009;98:643-50.

16. Spitzer RL, Kroenke K, Williams JB. Validation and utility of a self-report version of PRIME-MD: the PHQ primary care study. Primary Care Evaluation of Mental Disorders. Patient Health Questionnaire. JAMA. 1999;282:1737-44.

17. Kroenke K, Spitzer RL, Williams JB. The PHQ-9: validity of a brief depression severity measure. J Gen Intern Med. 2001;16:606-13.

18. Liu SI, Yeh ZT, Huang HC, Sun FJ, Tjung JJ, Hwang LC, et al. Validation of Patient Health Questionnaire for depression screening among primary care patients in Taiwan. Compr Psychiatry. 2011;52:96-101.

19. Pinto-Meza A, Serrano-Blanco A, Peñarrubia MT, Blanco E, Haro JM. Assessing depression in primary care with the PHQ-9: can it be carried out over the telephone? J Gen Intern Med. 2005;20:738-42.

20. Faith MS, Butryn M, Wadden TA, Fabricatore A, Nguyen AM, Heymsfield SB. Evidence for prospective associations among depression and obesity in population-based studies. Obes Rev. 2011;12:e438-53.

21. Albert MA, Danielson E, Rifai N, Ridker PM. Effect of statin therapy on C-reactive protein levels: the pravastatin inflammation/CRP evaluation (PRINCE): a randomized trial and cohort study. JAMA. 2001;286:64-70.

22. Pizzi C, Manzoli L, Mancini S, Bedetti G, Fontana F, Costa GM. Autonomic nervous system, inflammation and preclinical carotid atherosclerosis in depressed subjects with coronary risk factors. Atherosclerosis. 2010;212:292-8.

23. McCaffery JM, Frasure-Smith N, Dubé MP, Théroux P, Rouleau GA, Duan Q et al. Common genetic vulnerability to depressive symptoms and coronary artery disease: a review and development of candidate genes related to inflammation and serotonin. Psychosom Med. 2006;68:187-200.

24. de Jonge P, Rosmalen JG, Kema IP, Doornbos B, van Melle JP, Pouwer F, et al Psychophysiological biomarkers explaining the association between depression and prognosis in coronary artery patients: a critical review of the literature. Neurosci Biobehav Rev. 2010;35:84-90.

25. Dowlati Y, Herrmann N, Swardfager W, Liu H, Sham L, Reim EK, et al. A metaanalysis of cytokines in major depression. Biol Psychiatry. 2010;67:446-57. 